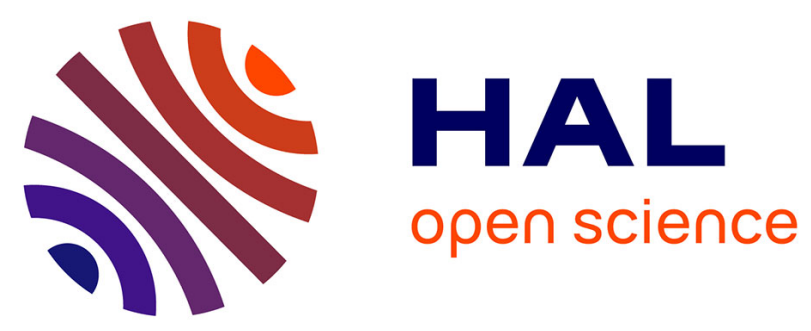

\title{
Conformational preferences and internal rotation of methyl butyrate by microwave spectroscopy
}

Alicia O Hernandez-Castillo, Chamara Abeysekera, Brian Hays, Isabelle Kleiner, Ha Vinh Lam Nguyen, Timothy S Zwier

\section{- To cite this version:}

Alicia O Hernandez-Castillo, Chamara Abeysekera, Brian Hays, Isabelle Kleiner, Ha Vinh Lam Nguyen, et al.. Conformational preferences and internal rotation of methyl butyrate by microwave spectroscopy. Journal of Molecular Spectroscopy, 2017, 337, pp.51-58. 10.1016/j.jms.2017.03.016 . hal-03183101

\section{HAL Id: hal-03183101 \\ https://hal.science/hal-03183101}

Submitted on 26 Mar 2021

HAL is a multi-disciplinary open access archive for the deposit and dissemination of scientific research documents, whether they are published or not. The documents may come from teaching and research institutions in France or abroad, or from public or private research centers.
L'archive ouverte pluridisciplinaire HAL, est destinée au dépôt et à la diffusion de documents scientifiques de niveau recherche, publiés ou non, émanant des établissements d'enseignement et de recherche français ou étrangers, des laboratoires publics ou privés. 


\title{
Conformational Preferences and Internal Rotation of Methyl Butyrate by Microwave Spectroscopy
}

\author{
Alicia O. Hernandez-Castillo ${ }^{1}$, Chamara Abeysekara ${ }^{1}$, Brian M. Hays ${ }^{1}$, \\ Isabelle Kleiner ${ }^{2}$, Ha Vinh Lam Nguyen ${ }^{2 *}$, and Timothy S. Zwier ${ }^{*}$.
}

${ }^{1}$ Department of Chemistry, Purdue University, 560 Oval Drive, West Lafayette, IN 47907, USA ${ }^{2}$ Laboratoire Interuniversitaire des Systèmes Atmosphériques (LISA), CNRS UMR 7583, Université Paris-Est Créteil, Université Paris Diderot, 61 avenue du Général de Gaulle, F-94010 Créteil cedex, France

\begin{abstract}
The broadband rotational spectrum of methyl butyrate from 8-18 GHz, recorded using a chirppulsed Fourier transform microwave (FTMW) spectrometer, was combined with high resolution FTMW measurements over the 2-26.5 GHz region to provide a comprehensive account of its microwave spectrum under jet-cooled conditions. Two low-energy conformers, one with a fully extended, heavy-atom planar antilanti structure (a,a), and the other with a gauche propyl chain $(\mathbf{g} \pm, \mathbf{a})$, were assigned in the spectrum. Torsional A/E splittings due to the internal rotation of the methoxy methyl group were resolved for both lower energy conformers, and were fitted using the program XIAM and BELGI, providing an estimate of the barrier to methyl internal rotation of $V_{3} \approx$ $420 \mathrm{~cm}^{-1}$. The conformational landscape of methyl butyrate occurs on a two-dimensional potential energy surface, which was mapped out by quantum chemical calculations at the B2PLYP$\mathrm{D} 3 \mathrm{BJ} /$ aug-cc-pVTZ level of theory. The low torsional barrier about the $\mathrm{C}-\mathrm{C}(=\mathrm{O}) \mathrm{O}$ bond leads to collisional removal of population originally in the $(\mathbf{a}, \mathbf{g} \pm)$ and $(\mathbf{g} \pm, \mathbf{g} \pm)$ minima into the $(\mathbf{a}, \mathbf{a})$ and $(\mathbf{g} \pm$,a) minima, respectively, during the cooling in the expansion. Analysis of experimental intensities in the spectrum provide percent populations downstream in the expansion of $41 \pm 4 \%$ (a,a), and $59 \pm 6 \%(\mathbf{g} \pm, \mathbf{a})$.
\end{abstract}

*To whom correspondence should be addressed. E-mail: lam.nguyen@lisa.u-pec.fr, zwier@purdue.edu

Key Words: methyl butyrate, rotational spectroscopy, large amplitude motions, conformational analysis

to be submitted to the Journal of Molecular Spectroscopy

18 pages, 3 Tables, 5 Figures 


\section{Introduction}

Methyl esters of fatty acids are important components of biologically-derived diesel fuel ("biodiesel"), produced by reacting vegetable oils or animal fats with an alcohol [1]. By comparison with petroleum diesel, which contains straight-chain alkanes, the alkyl chains in the fatty acids serving as a source of biodiesel are a mixture of straight-chain, branched-chain, and with partial unsaturation [2]. Not surprisingly, the combustion chemistry of fatty acid methyl esters will thus have reactions characteristic of the long alkyl or alkenyl "tail" and methyl ester "head" groups. While the alkyl chains are common to petroleum diesel, the presence of the ester functional group complicates the combustion chemistry of biodiesel, which needs to be investigated in some detail as sources for biodiesel become more common and begin to be incorporated into modern transportation fuels more pervasively.

Methyl butyrate or methyl butanoate, $\mathrm{CH}_{3}-\mathrm{CH}_{2}-\mathrm{CH}_{2}-\mathrm{C}(=\mathrm{O})-\mathrm{O}-\mathrm{CH}_{3}$, combines the methyl ester with an alkyl chain just long enough to capture some of the key features anticipated for the combustion chemistry of longer-chain methyl esters. As a result, methyl butyrate serves in combustion studies as a model system in which to study the ester group's combustion chemistry, despite its deficiencies in not fully capturing the chemistry of the longer alkyl chains fatty acid $[3,4]$. Beyond its role in combustion, methyl butyrate is interesting in its own right as a natural product, possessing a fruity odor similar to that of many natural-product methyl esters. It is widely used in perfumery as an odorant and food flavoring.

Methyl butyrate has an alkyl chain sufficiently long to support conformational isomers. As a result, whether in the context of combustion or detection by smell, the inherent conformational preferences of gas phase methyl butyrate are important to understand. Thus, in the present work, we describe a study of the title molecule by supersonic jet Fourier transform microwave (FTMW) spectroscopy, aided by quantum chemical calculations. Our goal is to determine the preferred conformations of methyl butyrate and their relative populations. Identifying the inherent conformational preferences of this model alkyl methyl ester is a necessary first step in subsequent studies of methyl butyrate's pyrolysis intermediates, as well as in understanding the molecular origin of its fruity odor.

Regarding the internal dynamics, we expected that torsional splittings arising from the internal rotation of the methoxy methyl group $\mathrm{C}(=\mathrm{O})-\mathrm{O}-\mathbf{C H} 3$ could be resolved, as this aspect of its chemical structure is similar to other methyl alkylates, such as methyl acetate [5] and methyl propionate [6], studied previously by some of the authors. On the other hand, the barrier to internal rotation, arisen from the second methyl group at the end of the alkyl chain (called the propyl methyl group), is estimated to be about $1100 \mathrm{~cm}^{-1}$; similar to that of the ethyl methyl group in ethyl acetate [7]. As we shall see, we are not able to fully resolve the splittings from this large amplitude motion with our spectrometers.

Some of us have recently introduced the technique of strong-field coherence breaking (SFCB) that combines excitation of the sample by broad-band chirped-pulse microwave excitation with multi-resonant single-frequency pulses to achieve selective detection of a set of microwave 
transitions due to one molecular component in a mixture, thereby aiding the analysis of spectra of such samples. In the context of conformational isomers, this previous study used methyl butyrate as example, showing how SFCB studies can identify the two observed conformers of methyl butyrate [8].

\section{Theoretical Calculations}

\subsection{Conformational Analysis}

Since the rotations of the two methyl groups in methyl butyrate do not create new conformations, the conformational landscape is completely defined by three dihedral angles $\varphi_{1}=$ $\angle\left(\mathrm{C}_{1}, \mathrm{C}_{2}, \mathrm{C}_{3}, \mathrm{C}_{4}\right), \varphi_{2}=\angle\left(\mathrm{C}_{2}, \mathrm{C}_{3}, \mathrm{C}_{4}, \mathrm{O}_{6}\right)$, and $\varphi_{3}=\angle\left(\mathrm{C}_{3}, \mathrm{C}_{4}, \mathrm{O}_{6}, \mathrm{C}_{7}\right)$, corresponding to the rotations about the $\mathrm{C}_{2}-\mathrm{C}_{3}, \mathrm{C}_{3}-\mathrm{C}_{4}$, and $\mathrm{C}_{4}-\mathrm{O}_{6}$ bonds (for atom numbering see Figure 1). The rotation about the $\mathrm{C}_{4}-\mathrm{O}_{6}$ bond by varying the dihedral angle $\varphi_{3}$ results in trans $\left(\varphi_{3}=180^{\circ}\right)$ and cis ester $\left(\varphi_{3}=0^{\circ}\right)$ conformations. As known from our previous investigations on other esters [7,9] and theoretical studies [10], only trans esters can be observed using the supersonic jet technique, where the rotational temperature is very low, approximately $2 \mathrm{~K}$, because cis esters are much higher in energy (often more than $25 \mathrm{~kJ} \cdot \mathrm{mol}^{-1}$, in methyl butyrate more than $35 \mathrm{~kJ} \cdot \mathrm{mol}^{-1}$ ). Hence, we only take the trans conformations into consideration in what follows.

To locate the conformational minima and to better understand the potential energy landscape, we thus calculated a relaxed two-dimensional potential energy surface (2D-PES) depending on $\varphi_{1}$ and $\varphi_{2}$. These angles were varied over a grid of $\left(\varphi_{1}, \varphi_{2}\right)$ points separated by $15^{\circ}$ while optimizing all other coordinates. DFT calculations were employed using the B2PLYP functional, including Grimme's dispersion correction [11] and a Becke-Johnson damping [12], with a correlation consistent basis set, aug-cc-pVTZ, using the program GAUSSIAN [13]. The result is shown in Figure 2. This functional and basis set were chosen because in recent studies of alkyl chains [1416], this level of theory has been shown to give excellent results for the relative energies of conformers. Four conformers of methyl butyrate were identified on the PES and subsequently reoptimized under full geometry optimizations at the MP2/6-311++G(d,p) level of theory. This level of theory was chosen for the geometries because it yields rotational constants which are in close agreement with experiment for many types of molecules [17-19]. The optimized geometries are illustrated in Figure 3 in order of their energies relative to that of the most stable conformer. Harmonic frequency calculations at both levels of theory confirm that these structures are true minima. We named the conformers according to the conformational arrangement of $\varphi_{1}$ and $\varphi_{2}$ dihedral angles. Using a for antiperiplanar conformations (corresponding torsional angles between $-150^{\circ}$ and $\left.+150^{\circ}\right)$, $\mathbf{g} \pm$ was used for gauche or synclinal conformations $\left( \pm 30^{\circ}\right.$ to $\left.\pm 90^{\circ}\right)$.

Only conformer $(\mathbf{a}, \mathbf{a})$ located at $\left(\varphi_{1}, \varphi_{2}\right)=\left(180.0^{\circ}, 180.0^{\circ}\right)$ has $C_{s}$ symmetry; all other conformers, $(\mathbf{g} \pm, \mathbf{a}),(\mathbf{g} \pm, \mathbf{g} \pm)$, and $(\mathbf{a}, \mathbf{g} \pm)$, possess $\mathrm{C}_{1}$ symmetry and exist in what are essentially enantiomeric pairs by virtue of the direction of curvature of the alkyl chain, though there are no chiral centers. The enantiomers have the same electronic energy and rotational constants, and therefore cannot be distinguished under our measurement conditions. Comparing the present 
nomenclature to that in Ref. [20], $(\mathbf{a}, \mathbf{a}),(\mathbf{g} \pm, \mathbf{a}),(\mathbf{g} \pm, \mathbf{g} \pm)$, and $(\mathbf{a}, \mathbf{g} \pm)$ are equivalent to $\mathbf{a a}, \mathbf{a M} / \mathbf{a P}$, $\mathbf{P P} / \mathbf{M M}$, and $\mathbf{M a} / \mathbf{P a}$ used in that work, respectively.

The global minimum conformer $(\mathbf{g} \pm, \mathbf{a})$ is calculated to occur at $\left(\varphi_{1}, \varphi_{2}\right)=\left(\mp 68.5^{\circ}, \pm 158.7^{\circ}\right)$ and has the propyl methyl group tilted out of the molecular frame spanned by the $\mathrm{C}_{4}, \mathrm{O}_{5}, \mathrm{O}_{6}, \mathrm{C}_{7}$ atoms that make up the methyl ester. Two other conformers, $(\mathbf{g} \pm, \mathbf{g} \pm)$ and $(\mathbf{a}, \mathbf{g} \pm)$, that are higher in energy, both have $\varphi_{2} \approx \pm 60^{\circ}$. The rotational constants $A, B, C$ for all conformers, their dipole moment components, and energies relative to that of conformer $(\mathbf{g} \pm, \mathbf{a})$ (including zero-point energy correction) are summarized in Table 1 . The atom positions in the principal axis system can be found in Table S-1 in the supplementary material.

It is known that in a supersonic expansion the gas undergoes collisional cooling which, depending on their barrier to isomerization, can affect the population of certain conformers and funnel the population down to lower energy minima [21]. In order to predict the conformer distribution prior to the expansion cooling, the fractional populations of each of the local minima, $F(i)=\frac{N_{i}}{N_{t o t}}=\frac{g_{i} \exp \left(-\frac{\Delta G(i)}{R T}\right)}{\sum_{i} g_{i} \exp \left(-\frac{\Delta G(i)}{R T}\right)}$, were calculated at $300 \mathrm{~K}$, where $\Delta G(\mathrm{i})$ is the Gibbs energy difference of minimum "i" relative to the global minimum at standard conditions, using the free energies predicted at B2PLYP-D3BJ/aug-cc-pVTZ and MP2/6-311++G(d,p) levels of theory. The results for the percent populations are given in Table 2.

\subsection{Basis Set Variation}

As mentioned in section 2.1, full geometry optimizations for the four conformers were first carried out at the MP2/6-311++G(d,p) and B2PLYP-D3BJ/aug-cc-PVTZ levels of theory. However, after the rotational spectrum had been assigned (see section 3.2. and section 4 on discussion), we recognized that the calculated rotational constants at the MP2/6-311++G(d,p) level differ by up to $9 \%$ from the experimentally determined values. At the B2PLYP-D3BJ/aug-ccpVTZ level of theory, these constants differ by less than $2.5 \%$ from experiment, a smaller but still significant difference. To explore further the level of theory that best matches experimental rotational constants, a series of MP2 and DFT calculations with different basis sets were carried out on the two assigned conformers. The calculated rotational constants are summarized in Table S-2 in the supplementary material.

\subsection{Internal Rotation}

The barrier to internal rotation of the methoxy methyl group was calculated for the lowest energy conformers, $(\mathbf{g} \pm, \mathbf{a})$ and $(\mathbf{a}, \mathbf{a})$, by rotating the methyl group about the $\mathrm{O}_{6}-\mathrm{C}_{7}$ bond. This was done by varying the dihedral angle $\varphi_{4}=\angle\left(\mathrm{C}_{4}, \mathrm{O}_{6}, \mathrm{C}_{7}, \mathrm{H}\right)$ in $10^{\circ}$ increments, while all other parameters were optimized at the same levels of theory previously mentioned in section 2.2. 


\section{Microwave spectroscopy}

\subsection{Experimental Details}

\subsubsection{Broadband spectrum}

The broadband rotational spectrum of methyl butyrate was recorded in the $8-18 \mathrm{GHz}$ frequency range using a chirped pulse FTMW spectrometer located at Purdue University. The instrument has been described in detail elsewhere [8]. A gas mixture of $1 \%$ methyl butyrate in $\mathrm{He}$ as the carrier gas at a backing pressure of $2.3 \mathrm{bar}$, was introduced into the chamber through a $1 \mathrm{~mm}$ nozzle orifice via a pulsed valve (Parker General, Series 9). The solenoid valve was triggered at $10 \mathrm{~Hz}$ repetition rate using a home-made driver. The experimental timing scheme allowed the collection of 25 molecular free induction decays (FIDs) per gas pulse. The molecular emission was phase coherently averaged in the time domain and digitized for $16 \mu \mathrm{s}$ with a $13 \mathrm{GHz}, 40 \mathrm{GS} / \mathrm{s}$ real-time digitizer (Guzik ADC6131), yielding an experimental resolution of $60 \mathrm{kHz}$. The corrected FID baseline was obtained using a custom Matlab routine. The data was filtered with a Kaiser-Bessel Digital filter and a Fast FT was performed to obtain the frequency domain spectrum. The broadband survey scan, which is shown in Figure 4, was used as an overview spectrum for the initial assignment (see section 3.2).

\subsubsection{High resolution measurements}

High resolution measurements were recorded in the frequency range from $2-26.5 \mathrm{GHz}$ using a molecular jet FTMW spectrometer described in Ref. [22]. A gas mixture containing 1\% methyl butyrate in helium at a total pressure of 1 bar was used for all measurements. All lines appear as doublets due to the Doppler effect. The molecular transition frequency is taken as the center frequency between these doublets. A typical spectrum for a high resolution measurement is also given in Figure 4. The estimated measurement accuracy is better than $2 \mathrm{kHz}$ for an isolated line [23]. However, due to the internal rotation of the propyl methyl group, many A species transitions are split into doublets and E species transitions into triplets. Sometimes, multiplets are found, which are difficult to assign. The splittings are up to $70 \mathrm{kHz}$ in magnitude.

\subsection{Spectral assignment}

\subsubsection{Conformer $(\mathbf{g} \pm, \mathbf{a})$}

As mentioned in the introduction, all rotational lines in the spectrum split into two torsional components of $\mathrm{A}$ and the $\mathrm{E}$ symmetry, due to the internal rotation of the methoxy methyl group. The Hamiltonian can be written as $H=H_{\text {rot }}+H_{c d}+H_{i}$, where $H_{\text {rot }}$ refers to the rigid rotor Hamiltonian, $H_{c d}$ is the Hamiltonian associated with the centrifugal part, and $H_{i}$ is the internal rotation of the methyl top [24].

In initial fitting, we neglected the last of these terms and treated methyl butyrate as a rigid rotor, considering only the unsplit A levels. The results of quantum chemical calculations in section 2.1 indicated four conformers within an energy range of only $3 \mathrm{~kJ} \mathrm{~mol}^{-1}$. We started the assignment with the lowest energy conformer, $(\mathbf{g} \pm, \mathbf{a})$ conformer. The microwave spectrum from 
8-18 GHz was calculated using the rotational constants predicted at the chosen levels of theory, and a preliminary assignment was done with the spfit/spcat suite of programs using a Watson-A reduced Hamiltonian [25], using the conformer specific transitions provided by the SFCB method, which is described in more detail in Ref. [8]. The SFCB tailored sequential chirp multipleselective excitation scheme (SFCB(TSC)/MSE) identified a set of 47 lines whose intensities were modulated more than $20 \%$ by the set of three single-frequency pulses. Refinement of the fit through inclusion of centrifugal distortion constants led to A-symmetry methyl rotor lines in the spectrum being reproduced to experimental accuracy in the broadband spectrum.

The rotational transition sets built off the $\mathrm{A}$ and $\mathrm{E}$ internal rotor levels have different nuclear spin symmetry, and therefore do not interconvert during the collisional cooling in the supersonic expansion. In principle, the SFCB method could be used to selectively modulate transitions due to either A or E methyl rotor levels, aiding in their assignment. However, due to their close proximity (typical splittings $<2 \mathrm{MHz}$ ), selective excitation of only A or only E levels under strongfield conditions were not possible. This is mainly due to power leakage of the high power, short (150 ns) coherence breaking pulses. With previous experiments we have found that by applying specific window functions to the excitation pulses this "cross-talk" can be minimized; however, a clean separation of A and E levels was not possible in methyl butyrate.

Characteristic patterns of transitions appeared close to those of the A levels, suggesting themselves as the E level components of the same rotational transitions. The program XIAM [26] was then used to predict the methyl rotor splittings of the E symmetry lines using the rotational constants and centrifugal distortion constants of the A levels as starting parameters. The barrier to hindered rotation of the methoxy methyl group was set to $420 \mathrm{~cm}^{-1}$, which is approximately the barrier found for methyl acetate [5] and methyl propionate [6]. The angles between the internal rotor axis and the principal axes were set based on the calculated geometry of conformer $(\mathbf{g} \pm, \mathbf{a})$ obtained by quantum chemical calculations at the B2PLYP-D3BJ/aug-cc-PVTZ level of theory. In so doing, the $\mathrm{E}$ level frequencies of many transitions were predicted accurately by the calculations, leading to their straightforward assignment.

Finally, we could assign $35 \mathrm{~A}$ and $32 \mathrm{E}$ lines, with a standard deviation of $29 \mathrm{kHz}$, in the 8-18 $\mathrm{GHz}$ spectrum broadband spectrum. Using the prediction from this fit, high resolution measurements were performed in the frequency range 2-26.5 GHz, leading to an expanded assignment of $53 \mathrm{~A}$ and $60 \mathrm{E}$ lines of $(\mathbf{g} \pm, \mathbf{a})$, and reducing the standard deviation on the fits to 4 $\mathrm{kHz}$. A fit using the program BELGI- $C_{1}$ [27], which can include more higher order terms, did not reduce the deviation significantly $(3.6 \mathrm{kHz})$. While the standard deviation is still twice the measurement accuracy, the additional splittings due to the propyl methyl rotor, which were not modeled, prevented further improvement. A list of all fitted transitions using the XIAM and the $B E L G I-C_{1}$ codes along with their residues is available in Table S-3 in the supplementary material. The BELGI- $C_{1}$ parameters in the Rho-Axis-Method [26] are summarized in Table S-4. 


\subsubsection{Conformer (a,a)}

Based on the initial SFCB measurements, 18 transitions due to a second conformer were identified. Based on the experience of some of us on linear alkyl acetates, it was known that a planar $\mathrm{C}_{\mathrm{s}}$ conformer was typically low enough in energy to be observed in their experimental spectra [28,29]. This is in agreement with the calculations in section 2.1 (B2PLYP-D3BJ/aug-ccpVTZ level of theory), where the (g士,a) and (a,a) conformers are very close in energy, while other two conformers are more than $2 \mathrm{~kJ} \cdot \mathrm{mol}^{-1}$ higher. Therefore, the next conformer we consider is (a,a). Noticing that the experimental conformer-specific transitions from SFCB(TSC)/MSE matched the pattern of the calculated 8-18 GHz microwave spectrum for this conformer, we proceeded to do a preliminary assignment of the A species as a rigid rotor. In the broadband spectrum, we were also able to resolve the splitting of the internal rotation of the methoxy methyl group for conformer (a,a), which were approximately $2 \mathrm{MHz}$ in the low-frequency region. Thus, we used the program XIAM to assign the transitions associated with the $\mathrm{E}$ internal rotor levels in the same manner as done for conformer $(\mathbf{g} \pm, \mathbf{a})$. In the broadband scan, a total of $32 \mathrm{~A}$ and $\mathrm{E} b$ type $R$-branch transitions with $K_{a}=0,1$ were assigned and fitted to a standard deviation of $41 \mathrm{kHz}$.

The assignment of higher $K_{a}$ transitions in (a,a) was more difficult than in the case of conformer $(\mathbf{g} \pm, \mathbf{a})$, because the $A$ rotational constant of conformer $(\mathbf{a}, \mathbf{a})$ is much larger and less well-constrained by the fit. Additional high-resolution scans in the region 20-23 GHz were carried out that detected transitions that could be assigned as $b$-type $Q$-branch transitions with $K_{a}=2 \leftarrow$ 1. In total, $42 \mathrm{~A}$ transitions and $43 \mathrm{E}$ transitions were assigned involving transitions up to $J=15$, with a standard deviation of $7.8 \mathrm{kHz}$ on the fit. The program BELGI-Cs [30] provided the same fit quality with somewhat better deviation of $5.2 \mathrm{kHz}$ using 13 parameters (see Table $\mathrm{S}-4$ in the supplementary material). We note that some $c$-type transitions involving $\mathrm{E}$ internal rotor levels were observable, which are also included in the frequency lists (Table S-3). It is known that for the E levels, such forbidden transitions can be observed, as previously found in e.g. ethyl acetate [7], vinyl acetate [30], and butadienyl acetate [9]. In the case of rigid rotors and the A species of molecules with internal rotation, the $K_{a} K_{c}$ pseudo quantum numbers can be used to derive the symmetry and thereby the selection rules. This is no longer true for the E species. Here the $K_{a} K_{c}$ quantum numbers just give the order of the energy levels and do not contain any symmetry information. Consequently, also by accident $c$-type transitions may be found even if no $c$ dipole moment component is present. Once again, many lines, especially those that belong to the $\mathrm{E}$ species, appear as triplets with splittings up to $70 \mathrm{kHz}$, which arise from the internal rotation of the propyl methyl group. As a result, also in this case, we did not attempt to reduce the standard deviation to measurement accuracy $(2 \mathrm{kHz})$, since this large amplitude motion was neglected in the analysis.

\section{Discussion}

\subsection{Barriers to Internal Rotation}

In the microwave spectrum of methyl butyrate recorded under jet-cooled conditions, rotational transitions due to two conformers were identified and assigned. A total of 85 rotational transitions 
belonging to the $\mathrm{C}_{\mathrm{s}}$ conformer (a,a) were fitted using three rotational constants, five quartic centrifugal distortion constants, the $V_{3}$ potential, and the angle $\delta$ between the internal rotor axis and the principal $a$ axis to a standard deviation of $7.8 \mathrm{kHz}$. Because only transitions in the torsional ground state are available, the rotational constant of the methyl rotor $F_{0}$ was fixed at $158 \mathrm{GHz}$, corresponding to a moment of inertia of $I_{\alpha}=3.2 \mathrm{u}^{2}$, to reduce the very strong correlation with $V_{3}$. For the more stable $\mathrm{C}_{1}$ conformer $(\mathbf{g} \pm \mathbf{a})$, we use the same set of parameters to fit 113 rotational lines to $4 \mathrm{kHz}$. In this case, also the polar coordinate $\varepsilon$, which describes the angle between the $b$ axis and the projection of the internal rotor axis onto the $b c$-plane, was determined. The torsional barrier of the methoxy methyl group extracted from the model is essentially the same in both assigned conformers, which is approximately $420 \mathrm{~cm}^{-1}$. We conclude that the electronic and steric environment around the ester methyl group is similar in both conformers [32]. These values are also very similar to those obtained for methyl acetate $\left(424.581(56) \mathrm{cm}^{-1}\right)$ [5] and methyl propionate $\left(429.324(23) \mathrm{cm}^{-1}\right)$ [6]. We surmise on that basis that the length and conformation of the alkyl chain on the opposite side of the ester group does not affect significantly the internal rotation of the methoxy methyl group.

\subsection{Torsional Surface and Conformational Preferences}

The calculated 2D-PES involving the two flexible torsional coordinates $\left(\varphi_{1}, \varphi_{2}\right)$ shown in Figure 2 serves to pictorialize the inherent conformational preferences of methyl butyrate. This minimalist fatty acid surrogate incorporates an alkyl chain of sufficient length to produce a total of seven low-lying conformational minima: the single (a,a) minimum, and three enantiomeric pairs $(\mathbf{g} \pm \mathbf{a}),(\mathbf{g} \pm, \mathbf{g} \pm)$, and $(\mathbf{a}, \mathbf{g} \pm)$. In the pure alkyl chain analog pentane, a similar set of two torsional coordinates would give rise to a total of nine minima, with each torsion occurring in a three-fold potential. In methyl butyrate, the two "missing" minima are in the upper left and lower right corners of the 2D surface in Figure 2, associated with the $(\mathbf{g} \pm, \mathbf{g} \mp)$ pair.

Figure 5 presents three minimum-energy pathways along the 2D surface. Figure 5(a) shows a cut along $\varphi_{1}$ through the (a,a) and $(\mathbf{g} \pm, \mathbf{a})$ minima. These minima are nearly isoenergetic, and contain all the observed population in this corridor at the nominally "anti" $\varphi_{2}$ configuration, which places the first two carbon atoms of the propyl chain near the plane of the ester group. The barriers to hindered rotation of the alkyl chain about $\varphi_{1}$ are more than $10 \mathrm{~kJ} \cdot \mathrm{mol}^{-1}\left(900 \mathrm{~cm}^{-1}\right)$.

The other two minimum energy pathways involve motion primarily along the $\varphi_{2}$ coordinate. The 2D surface is much softer along $\varphi_{2}$ than $\varphi_{1}$, creating long troughs along this coordinate. Figure 5 (b) is the minima energy pathway nominally associated with the gauche propyl chain conformations ( $\mathbf{g}+$ or $\mathbf{g}-)$. As previously mentioned, the $(\mathbf{g} \pm, \mathbf{g} \mp)$ configurations are not minima and therefore only two minima are present along this pathway.

A slice of the $2 \mathrm{D}$ surface along $\varphi_{2}$ with the propyl chain in the fully-extended "anti" configuration $\left(\varphi_{1}=180^{\circ}\right)$ is shown in Figure 5(c). Its shape is very much like that involving the ethyl group in methyl propionate [6], with a single deep (a,a) minimum and two secondary minima near the perpendicular configuration, whose exact location and barrier height are sensitive to the level of theory employed. 
The (g士,a) minima has the $\mathrm{C}_{2}-\mathrm{C}_{3}$ bond nearly in-plane (out-of-plane tilt angle of about $15^{\circ}$ ) on the oxygen side of the $\mathrm{C}=\mathrm{O}$ group, with the terminal methyl group of the alkyl chain nearly perpendicular to that plane. It is noteworthy that this configuration places one of the $\mathrm{CH}$ bonds of $\mathrm{C}(2)$ so that it interacts with the carbonyl oxygen, forming a weak $\mathrm{CH} \cdots \mathrm{O}=\mathrm{C}$ hydrogen bond. In so doing, it forms a five-membered $\mathrm{H}$-bonded ring, much as occurs in peptides between adjacent $\mathrm{NH}$ and $\mathrm{C}=\mathrm{O}$ groups in $\beta$-sheet secondary structures [33]. A second, less-stable pair of minima at $(\mathbf{g} \pm, \mathbf{g} \pm$ ) swings the propyl group around to the ester oxygen side of the structure, where it takes on a configuration closer to that of a free alkyl chain, as shown in Figure 5(c).

The shape of the PES presented in Figure 2 and the potential energy curves in Figure 5 also helps explain why only two of the four stable conformational minima are observed downstream in the expansion. The rotational temperature was determined with a Boltzmann plot by using the relationship between the integrated line intensities $(\mathrm{W})$ and corresponding lower state energy $\left(\mathrm{E}_{1}\right)$, see supplementary material (Figure S-1) [34]. Interestingly, best-fits to the rotational intensity patterns consistently led to slightly different rotational temperatures for the two conformers $(0.86$ $\pm 0.19 \mathrm{~K}$ vs. $0.35 \pm 0.08 \mathrm{~K}$ ), indicating some conformer specificity to the collisional cooling, with conformer (a,a) more efficiently cooled than $(\mathbf{g} \pm, \mathbf{a})$.

We used the experimental rotational temperatures and the relationship between experimental integrated line intensities and column densities to obtain fractional abundances [35]. The percent abundances at our experimental conditions were $41 \pm 4 \%$ (a,a) and $59 \pm 6 \%$ (g士,a) (Table 2). Based on the experimental signal-to-noise ratio, we were also able to place upper bounds on the percent populations of $(\mathbf{g} \pm, \mathbf{g} \pm$ ) and $(\mathbf{a}, \mathbf{g} \pm$ ) of less than $0.1 \%$. By comparison, as Table 2 summarizes, using the calculated relative free energies of the four conformers at the pre-expansion temperature $(300 \mathrm{~K})$, the percent populations of the $(\mathbf{g} \pm, \mathbf{g} \pm)$ and $(\mathbf{a}, \mathbf{g} \pm)$ conformers are predicted to be within a factor of $0.3-0.6$ of the population of the most abundant $(\mathbf{g} \pm, \mathbf{a})$ conformer, and therefore should have been easily detectable.

However, there are many examples in the literature of collisional removal of the population of secondary minima during the supersonic expansion process if the barrier to isomerization to a lower-energy minimum is small compared to the average internal energy initially available to the conformer [14]. Indeed, as Figure 5 shows, the barrier separating the (a,g \pm ) from the (a,a) well is predicted to be only $370 \mathrm{~cm}^{-1}\left(4.4 \mathrm{~kJ} \cdot \mathrm{mol}^{-1}\right)$, a value small enough that the $(\mathbf{a}, \mathbf{g} \pm)$ population can be completely transferred into (a,a) during the collisional cooling in the expansion. Similarly, the barrier separating $(\mathbf{g} \pm, \mathbf{g} \pm)$ from $(\mathbf{g} \pm \mathbf{a})$ is $602 \mathrm{~cm}^{-1}\left(7.2 \mathrm{~kJ} \cdot \mathrm{mol}^{-1}\right)$, significantly smaller than the average internal energy available to the conformers prior to the expansion $\left(\mathrm{E}_{\mathrm{ave}} \approx 1900 \mathrm{~cm}^{-1}\right)$. The $\mathrm{RRKM}$ rate constant for the $(\mathbf{g} \pm, \mathbf{g} \pm) \rightarrow(\mathbf{g} \pm, \mathbf{a})$ isomerization at this initial energy is $\mathrm{k}\left(\mathrm{E}_{\mathrm{ave}}\right) \approx 1 \times 10^{8}$ $\mathrm{s}^{-1}$, a rate sufficient to enable collisional relaxation into the $(\mathbf{g} \pm, \mathbf{a})$ minimum. In contrast, the barriers separating the $(\mathbf{g} \pm, \mathbf{a})$ and $(\mathbf{a}, \mathbf{a})$ minima are $928 \mathrm{~cm}^{-1}\left(11.1 \mathrm{~kJ} \cdot \mathrm{mol}^{-1}\right)$, with an isomerization rate of approximately $1 \times 10^{6} \mathrm{~s}^{-1}$, so that population initially residing in one of the vertical troughs is incapable of isomerizing into the other on the timescale of the cooling, and is thus trapped in the $(\mathbf{a}, \mathbf{a})$ or $(\mathbf{g} \pm, \mathbf{a})$ wells. 
We thus propose that the final population in the (a,a) well should be the sum of the room temperature populations in the (a,a) and (a,g $\mathbf{a})$ wells $(38 \%)$, while the final $(\mathbf{g} \pm, \mathbf{a})$ population is the sum of the initial $(\mathbf{g} \pm, \mathbf{g} \pm)$ and $(\mathbf{g} \pm, \mathbf{a})$ populations. The predictions of the B2PLYP-D3BJ calculations (Table 2$)$ are in excellent agreement with experiment $((\mathbf{a}, \mathbf{a})+(\mathbf{a}, \mathbf{g} \pm)=38 \%$ calculated vs. $41 \%$ experiment; $(\mathbf{g} \pm, \mathbf{g} \pm)+(\mathbf{g} \pm \mathbf{a})=62 \%$ calculated vs. $59 \%$ experiment $)$. This check provides evidence that the B2PLYP-D3BJ/aug-cc-pVTZ relative energies are more accurate than the standard MP2/6-311++G(d,p) level of theory (Table 2) often used in microwave studies.

\subsection{Rotational Constants and Calculated Geometries}

For the $\mathrm{C}_{\mathrm{s}}$ conformer $(\mathbf{a}, \mathbf{a})$, the rotational constants calculated using a range of levels of theory are in close agreement with the rotational constants derived from experiment, with deviations of less than $1 \%$ typical. This level of agreement is sufficiently close that the identification and assignment of transitions to a particular conformer is greatly facilitated.

However, the rotational constants of the $\mathrm{C}_{1}$ conformer $(\mathbf{g} \pm, \mathbf{a})$ obtained at the widely used MP2/6-311++G(d,p) level of theory are in much poorer agreement than this, with a deviation between experiment and theory of almost $9 \%$ in the $A$ rotational constant. This is a likely consequence of the relatively flat-bottomed potential along the $\varphi_{2}$ dihedral angle, which makes the optimized structure sensitive to the shape of this well and thus the level of theory. Indeed, it is in just such circumstances that the SFCB method is of use in identifying a set of transitions that can reliably be associated with a single conformer, despite having poor agreement with the predictions of calculation, as was done for methyl butyrate.

Since microwave data on larger molecules rely heavily on a close correspondence between calculated and experimental rotational constants, it is worth considering further the reason for the discrepancy. We carried out geometry optimizations using a range of computational methods and basis sets, as is summarized in Table S-2 in the supplementary material. We found that the $A$ rotational constant of conformer $(\mathbf{g} \pm, \mathbf{a})$ varies widely with level of theory, while it stays quite consistent in the case of conformer (a,a). More specifically, while the dihedral angle $\varphi_{1}$ varies by no more than $1^{\circ}$ (between $69^{\circ}$ and $70^{\circ}$ ), the $\varphi_{2}$ dihedral angle varies from $146^{\circ}$ to $169^{\circ}$. Furthermore, initially, we anticipated that the tilt angle $\Theta=180^{\circ}-\left|\varphi_{2}\right|$ of the $C(2)$ carbon atom of the ethyl group relative to the $\mathrm{C}_{3}-\mathrm{C}_{4}-\mathrm{O}_{6}$ plane would be near zero degrees, so that only the propyl methyl group would be out of plane. However, the results in Table S-2a show that this is not the case at all levels of theory tested. This type of symmetry breaking has frequently occurred in quantum chemical calculations on other esters previously studied, for example in ethyl pivalate [36] and ethyl valerate [37]. Based on the results in Table S-2a, we found that the largest deviations between calculated and experimental values are observed when diffuse functions are included in the MP2 calculations, which produce tilt angles $\Theta$ near $30^{\circ}$. If the diffuse functions are omitted, calculations with the MP2 method provide the same quality as those with other methods such as B2PLYP-D3BJ/aug-cc-PVTZ. In those cases, $\Theta$ is between 14 and $22^{\circ}$.

The most likely cause of this discrepancy between experiment and theory is the large intramolecular basis-set superposition error that plagues MP2 calculations without sufficient 
diffuse basis functions [38,39]. For the basis set sizes explored in this work, addition of the diffuse functions decreases the agreement with experiment, but their removal reaches the correct answer for the wrong reason. It is noteworthy that, at least in this case, the MP2 calculations also do a poorer job than dispersion-corrected DFT in accounting for the observed relative populations of the conformers, as shown in Table $2[40,41]$.

\section{Conclusions}

The microwave spectrum of methyl butyrate $\left(\mathrm{CH}_{3}-\mathrm{CH}_{2}-\mathrm{CH}_{2}-(\mathrm{C}=\mathrm{O})-\mathrm{O}-\mathrm{CH}_{3}\right)$ was measured over the 2-26 GHz range, leading to the identification and assignment of its two lowest-energy conformers. As a short-chain analog of the methyl esters of fatty acids, the conformational preferences of methyl butyrate shed light on how the first few members of longer alkyl chains are likely to configure relative to the methyl ester. A conformer in which the alkyl chain is a fullyextended, heavy-atom-planar $\mathrm{C}_{\mathrm{s}}$ symmetry structure, labeled as (a,a), has $41 \pm 4 \%$ of the population downstream in the expansion. The most abundant conformer $(\mathbf{g} \pm, \mathbf{a})$, with $59 \pm 6 \%$ of the population, has the methyl group at the end of the propyl chain preferentially on the carbonyl oxygen side of the molecule, extending nearly perpendicular to the plane of the ester group.

Both conformers have A/E splittings in the microwave spectrum due to the methyl ester that could be fitted within a simple 1D hindered rotor model to obtain three-fold barriers to methyl internal rotation of about $420 \mathrm{~cm}^{-1}$. The calculations indicate that the preferred orientation for the methyl group has one $\mathrm{CH}$ in the plane of the ester group, on the ether oxygen side. Finally, calculation of the full 2D torsional surface for the propyl chain provided an explanation for the lack of observation of two other low-lying conformers, $(\mathbf{g} \pm, \mathbf{g} \pm$ ) and $(\mathbf{a}, \mathbf{g} \pm)$, anticipated to have population in a $300 \mathrm{~K}$ distribution. The small barriers separating these wells from the (g士a) and (a,a) minima, respectively, provided low-energy pathways for population transfer to the lower energy minima during the collisional cooling process.

\section{Acknowledgement}

AHC, CA, BMH, and TSZ gratefully acknowledge support for this research from the Department of Energy Basic Energy Sciences, Chemical Sciences Division under Grant No. DE-FG0296ER14656. IK and HVLN thank M.Sc. Anja Hermanns, M.Sc. Lynn Ferres, and Dipl.-Chem. Ba Thanh Dang for their contributions during their research projects. The Faculty of Sciences and Technology of the University Paris-Est Créteil is acknowledged for research support.

\section{References}

[1] X. Li, X. Xu, X. You, D.G. Truhlar, J. Phys. Chem. A 120 (2016) 4025-4036.

[2] K. Kohse-Hoinghaus, P. Oswald, T.A. Cool, T. Kasper, N. Hansen, F. Qi, C.K. Westbrook, P.R. Westmoreland, Angew. Chem. Int. Ed. 49 (2010) 3572-3597.

[3] S. Gail, M.J. Thomson, S.M. Sarathy, S.A. Syed, P. Dagaut, P. Diévart, A.J. Marchese, F.L. Dryer, Proc. Combust. Inst. 31 (2007) 305-311.

[4] C.K. Westbrook, W.J. Pitz, H.J. Curran, J. Phys. Chem. A 110 (2006) 6912-6922.

[5] H.V.L. Nguyen, I. Kleiner, S.T. Shipman, Y. Mae, K. Hirose, S. Hatanaka, K. Kobayashi, J. Mol. Spectrosc. 299 (2014) 17-21. 
[6] H.V.L. Nguyen, W. Stahl, I. Kleiner, Mol. Phys. 110 (2012) 2035-2042.

[7] D. Jelisavac, D.C. Cortés-Gómez, H.V.L. Nguyen, L.W. Sutikdja, W. Stahl, I. Kleiner, J. Mol. Spectrosc. 257 (2009) 111-115.

[8] A.O. Hernandez-Castillo, C. Abeysekera, B.M. Hays, T.S. Zwier, J. Chem. Phys. 145 (2016) 114203.

[9] A. Jabri, V. Van, H.V.L. Nguyen, W. Stahl, I. Kleiner, ChemPhysChem 17 (2016) 26602665.

[10] M. Oki, H. Nakanishi, Bull. Chem. Soc. Japan 43 (1970) 2558-2566.

[11] S. Grimme, J. Chem. Phys. 124 (2006) 034108.

[12] S. Grimme, S. Ehrlich, L. Goerigk, J. Comput. Chem. 32 (2011) 1456-1465.

[13] M.J. Frisch, G.W. Trucks, H.B. Schlegel, G.E. Scuseria, M.A. Robb, J.R. Cheeseman, G. Scalmani, V. Barone, B. Mennucci, G. A. Petersson, H. Nakatsuji, M. Caricato, X. Li, H. P. Hratchian, A.F. Izmaylov, J. Bloino, G. Zheng, J.L. Sonnenberg, M. Hada, M. Ehara, K. Toyota, R. Fukuda, J. Hasegawa, M. Ishida, T. Nakajima, Y. Honda, O. Kitao, H. Nakai, T. Vreven, J.A. Montgomery, Jr., J.E. Peralta, F. Ogliaro, M. Bearpark, J.J. Heyd, E. Brothers, K.N. Kudin, V.N. Staroverov, R. Kobayashi, J. Normand, K. Raghavachari, A. Rendell, J.C. Burant, S.S. Iyengar, J. Tomasi, M. Cossi, N. Rega, J.M. Millam, M. Klene, J.E. Knox, J.B. Cross, V. Bakken, C. Adamo, J. Jaramillo, R. Gomperts, R.E. Stratmann, O. Yazyev, A.J. Austin, R. Cammi, C. Pomelli, J.W. Ochterski, R.L. Martin, K. Morokuma, V.G. Zakrzewski, G.A. Voth, P. Salvador, J.J. Dannenberg, S. Dapprich, A.D. Daniels, O. Farkas, J.B. Foresman, J.V. Ortiz, J. Cioslowski, D.J. Fox, Gaussian 09, Revision A.02, Gaussian, Inc., Wallingford CT, 2009.

[14] D. Zhang, S. Bocklitz, T.S. Zwier, J. Phys. Chem. A 120 (2016) 55-67.

[15] N.O.B. Lüttschwager, M.A. Suhm, Soft Matter 10 (2014) 4885-4901.

[16] D.P. Tabor, D.M. Hewett, S. Bocklitz, J.A. Korn, A.J. Tomaine, A.K. Ghosh, T.S. Zwier, E.L. Sibert, J. Chem. Phys. 144 (2016) 224310.

[17] Y. Zhao, W. Stahl, H.V.L. Nguyen, Chem. Phys. Lett. 545 (2012) 9-13.

[18] V. Van, W. Stahl, H.V.L. Nguyen, Phys. Chem. Chem. Phys. 17 (2015) 32111-32114.

[19] L.W. Sutikdja, D.. Jelisavac, W.. Stahl, I.. Kleiner, Mol. Phys. 110 (2012) 2883-2893.

[20] L.W. Sutikdja, W. Stahl, V. Sironneau, H.V.L. Nguyen, I. Kleiner, Chem. Phys. Lett. 663 (2016) 145-149.

[21] G.M. Florio, R.A. Christie, K.D. Jordan, T.S. Zwier, J. Am. Chem. Soc. 124 (2002) 1023610247.

[22] J.-U. Grabow, W. Stahl, H. Dreizler, Rev. Sci. Instrum. 67 (1996) 4072-4084.

[23] J.-U. Grabow, W. Stahl, Z. Naturforsch. 45a (1990) 1043-1044.

[24] I. Kleiner, J. Mol. Spectrosc. 260 (2010) 1-18.

[25] H.M. Pickett, J. Mol. Spectrosc. 377 (1991) 371-377.

[26] H. Hartwig, H. Dreizler, Z. Naturforsch, 51a, (1996) 923-932.

[27] I. Kleiner, J.T. Hougen, J. Chem. Phys. 119 (2003) 5505-5509.

[28] T. Attig, L.W. Sutikdja, R. Kannengießer, I. Kleiner, W. Stahl, J. Mol. Spectrosc. 284-285 (2013) 8-15.

[29] T. Attig, R. Kannengießer, I. Kleiner, W. Stahl, J. Mol. Spectrosc. 290 (2013) 24-30.

[30] J.T. Hougen, I. Kleiner, M. Godefroid, J. Mol. Spectrosc. 163 (1994) 559-586.

[31] H.V.L. Nguyen, A. Jabri, V. Van, W. Stahl, J. Phys. Chem. A 118 (2014) 12130-12136.

[32] A.J. Shirar, D.S. Wilcox, K.M. Hotopp, G.L. Storck, I. Kleiner, B.C. Dian, J. Phys. Chem. A 114 (2010) 12187-12194. 
[33] H. Fricke, A. Funk, T. Schrader, M. Gerhards, J. Am. Chem. Soc. 130 (2008) 4692-4698.

[34] J.M. Oldham, C. Abeysekera, B. Joalland, L.N. Zack, K. Prozument, I.R. Sims, B. Park, R.W. Field, A.G. Suits, J. Chem. Phys. 141 (2014) 154202.

[35] C. Abeysekera, B. Joalland, N. Ariyasingha, L.N. Zack, I.R. Sims, R.W. Field, A.G. Suits, J. Phys. Chem. Lett. 6 (2015) 1599-1604.

[36] H. Mouhib, Y. Zhao, W. Stahl, J. Mol. Spectrosc. 261 (2010) 59-62.

[37] H. Mouhib, W. Stahl, ChemPhysChem 13 (2012) 1297-1301.

[38] R.M. Balabin, Mol. Phys. 109 (2011) 943-953.

[39] L.F. Holroyd, T. van Mourik, Chem. Phys. Lett. 442 (2007) 42-46.

[40] M. Orio, D.A. Pantazis, F. Neese, Photosynth. Res. 102 (2009) 443-453.

[41] J.-D. Chai, S.-P Mao, Chemical Physics Letters, 538 (2012) 121-125. 


\section{Tables}

Table 1. The rotational constants $A, B, C$ (in GHz), dipole moment components $\mu_{a}, \mu_{b}, \mu_{c}$ (in Debye), and relative energies $E_{\text {rel. }}$ (in $\mathrm{kJ} \cdot \mathrm{mol}^{-1}$ ) of the indicated conformers of methyl butyrate calculated at the MP2/6-311++G(d,p) and B2PLYP-D3BJ/aug-cc-PVTZ levels of theory. The dipole moment components are with respect to the principal axes of inertia; signs refer to coordinates given in the supplementary materials. All energies are electronic energies including vibrational zero-point correction and are relative to the lowest energetic conformer $(\mathbf{g} \pm, \mathbf{a})$ with its absolute energy of $E+Z P E=-345.988667$ and -346.734269 Hartrees at the MP2 and B2PLYP levels of theory, respectively.

\begin{tabular}{lccccccccc}
\hline & \multicolumn{4}{c}{ MP2/6-311++G(d,p) } & \multicolumn{3}{c}{ B2PLYP-D3BJ/aug-cc-PVTZ } \\
\hline Conf. & $(\mathbf{g} \pm, \mathbf{a})$ & $(\mathbf{a}, \mathbf{a})$ & $(\mathbf{g} \pm, \mathbf{g} \pm)$ & $(\mathbf{a}, \mathbf{g} \pm)$ & $(\mathbf{g} \pm, \mathbf{a})$ & $(\mathbf{a}, \mathbf{a})$ & $(\mathbf{g} \pm, \mathbf{g} \pm)$ & $(\mathbf{a}, \mathbf{g} \pm)$ \\
\hline$A$ & 5.521 & 8.339 & 4.251 & 6.134 & 5.935 & 8.388 & 4.323 & 6.307 \\
$B$ & 1.452 & 1.198 & 1.703 & 1.306 & 1.426 & 1.198 & 1.667 & 1.291 \\
$C$ & 1.414 & 1.076 & 1.572 & 1.180 & 1.351 & 1.076 & 1.551 & 1.174 \\
$\mu_{a}$ & 0.239 & -0.067 & -0.606 & 0.646 & -0.386 & 0.103 & -0.503 & -0.511 \\
$\mu_{b}$ & 1.621 & -1.756 & -1.792 & -1.963 & 1.554 & -1.708 & -1.790 & -1.904 \\
$\mu_{c}$ & -0.593 & -0.001 & -1.073 & -0.305 & 0.557 & 0.000 & -0.950 & -0.245 \\
$E_{\text {rel. }}$ & 0 & 0.96 & 1.15 & 2.51 & 0 & 0.13 & 2.54 & 2.63 \\
\hline \hline
\end{tabular}


Table 2: Comparison between theoretical and experimental Boltzmann distribution. The theoretical values were calculated using B2PLYP-D3BJ/aug-cc-pVTZ level of theory.

\begin{tabular}{cccc}
\hline & $\begin{array}{c}\text { Experimental } \\
\text { abundance / \% }\end{array}$ & \multicolumn{2}{c}{ Boltzmann Population Ratio (300 K) / \% } \\
\cline { 3 - 4 } & & B2PLYP-D3BJ/aug-cc-pVTZ & MP2/6-311++G(d,p) \\
\hline$(\mathbf{a}, \mathbf{a})$ & $41(4)$ & 21.7 & 14.6 \\
\hline$(\mathbf{g} \pm, \mathbf{a})$ & $59(6)$ & 45.8 & 42.8 \\
\hline$(\mathbf{g} \pm \mathbf{g} \pm)^{\mathrm{b}}$ & $<0.1$ & 16.5 & 27.0 \\
\hline$(\mathbf{a}, \mathbf{g} \pm)^{\mathrm{b}}$ & $<0.1$ & 15.9 & 15.6 \\
\hline
\end{tabular}

${ }^{a}$ Experimental abundances of $(\mathbf{a}, \mathbf{a})$ and $(\mathbf{g} \pm, \mathbf{a})$ were derived using the experimental temperatures from the Boltzmann plot (Figure S-1). Errors denote $1 \sigma$ uncertainty in the last digit.

${ }^{\mathrm{b}}$ Calculated percent populations for these non-observed conformers are based on an average of the rotational temperatures of the observed conformers $(0.595 \mathrm{~K})$. The percentages for all conformers except (a,a) were multiplied by a factor of 2 to account for the enantiomeric pairs. 
Table 3. Molecular parameters of conformers $(\mathbf{g} \pm, \mathbf{a})$ and $(\mathbf{a}, \mathbf{a})$ of methyl butyrate obtained from the best fits to the frequencies.

\begin{tabular}{llll}
\hline Par. $^{\mathrm{a}}$ & $\mathrm{Unit}$ & \multicolumn{1}{c}{$(\mathbf{g} \pm, \mathbf{a})$} & \multicolumn{1}{c}{$(\mathbf{a}, \mathbf{a})$} \\
\hline$A$ & $\mathrm{GHz}$ & $6.05932256(59)$ & $8.3497789(17)$ \\
$B$ & $\mathrm{GHz}$ & $1.42134885(29)$ & $1.19776059(23)$ \\
$C$ & $\mathrm{GHz}$ & $1.33334892(33)$ & $1.07654601(21)$ \\
$\Delta_{J}$ & $\mathrm{kHz}$ & $0.6018(37)$ & $0.0493(11)$ \\
$\Delta_{J K}$ & $\mathrm{kHz}$ & $-5.930(15)$ & $0.440(17)$ \\
$\Delta_{K}$ & $\mathrm{kHz}$ & $51.785(52)$ & $8.78(33)$ \\
$\delta_{J}$ & $\mathrm{kHz}$ & $-0.13468(33)$ & $0.00478(14)$ \\
$\delta_{K}$ & $\mathrm{kHz}$ & $12.289(57)$ & \\
$F_{0}{ }^{\mathrm{b}}$ & $\mathrm{GHz}$ & 158. & 158. \\
$I_{\alpha}^{\mathrm{c}}$ & $\mathrm{uA} \AA^{2}$ & 3.199 & 3.199 \\
$V_{3}$ & $\mathrm{~cm}{ }^{-1}$ & $419.447(59)$ & $420.155(71)$ \\
$s^{\mathrm{d}}$ & & 34.28326 & 33.74499 \\
$\angle(i, a)$ & $\circ$ & $30.528(54)$ & $19.903(81)$ \\
$\angle(i, b)$ & $\circ$ & $59.521(98)$ & $70.097(81)$ \\
$\angle(i, c)$ & $\circ$ & $88.4(20)$ & $90.0^{\mathrm{e}}$ \\
$\sigma^{\mathrm{f}}$ & $\mathrm{kHz}$ & 4.0 & 7.8 \\
$\mathrm{~N}_{\mathrm{A}} / \mathrm{NE}^{\mathrm{g}}$ & & $53 / 60$ & $42 / 43$ \\
\hline
\end{tabular}

${ }^{a}$ All parameters refer to the principal axis system. Watson's A reduction in the $\mathrm{I}^{\mathrm{r}}$ representation was used. ${ }^{\mathrm{b}}$ Fixed to a reasonable value often obtained for a methyl group (see text). ${ }^{\mathrm{c}}$ Moment of inertia of the internal rotor, derived from its rotational constant $F_{0} .{ }^{\mathrm{d}}$ Reduced barrier $s=4 V_{3} / 9 F$. Fixed due to symmetry. ${ }^{\mathrm{f}}$ Standard deviation of the fit. ${ }^{\mathrm{g}}$ Number of the $\mathrm{A}\left(\mathrm{N}_{\mathrm{A}}\right)$ and $\mathrm{E}$ species lines $\left(\mathrm{N}_{\mathrm{E}}\right)$. 


\section{Figures}

Figure 1: Optimized geometries of the assigned conformers of methyl butyrate calculated at the B2PLYP-D3BJ/aug-cc-pVTZ level of theory. Upper trace: front view, lower trace: view along the $\mathrm{C}_{4}-\mathrm{O}_{5}$ bond. A tilt angle of the ethyl group $\left(\Theta=180^{\circ}-\left|\varphi_{2}\right|=15^{\circ}\right)$, relative to the $\mathrm{C}_{4}, \mathrm{O}_{5}, \mathrm{O}_{6}, \mathrm{C}_{7}$ plane, was calculated for the more stable conformer $(\mathbf{g} \pm, \mathbf{a})$ (lower left).
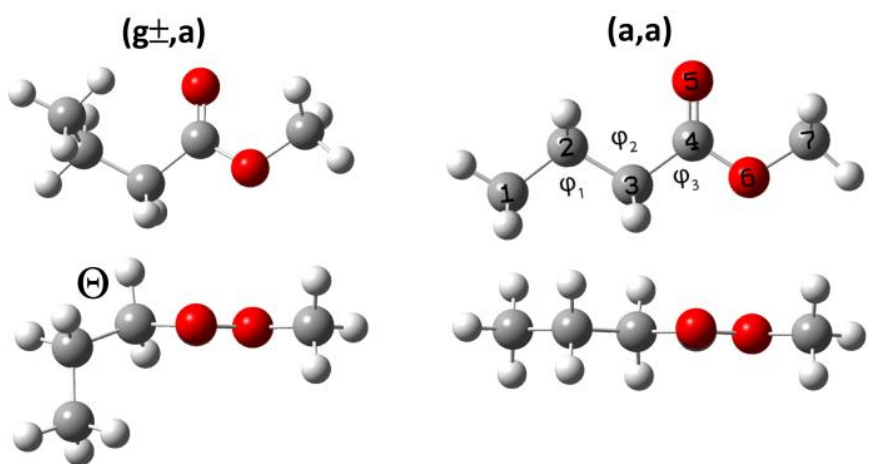

Figure 2: Potential energy surface of methyl butyrate for conformational analysis. Here four stable conformers were found: $1=(\mathbf{g}+\mathbf{a}), 2=(\mathbf{g}+, \mathbf{g}+), 3=(\mathbf{a}, \mathbf{g}-), 4=(\mathbf{a}, \mathbf{a}), 5=(\mathbf{a}, \mathbf{g}+), 6=(\mathbf{g}-, \mathbf{g}-), 7=$ (g-,a). Conformer 1 and 7, 2 and 6, and 3 and 5 are enantiomers. This surface was obtained by rotating the dihedral angles $\varphi_{1}=\angle\left(\mathrm{C}_{1}, \mathrm{C}_{2}, \mathrm{C}_{3}, \mathrm{C}_{4}\right)$ (rotation about the $\mathrm{C}_{2}-\mathrm{C}_{3}$ bond) and $\varphi_{2}=$ $\angle\left(\mathrm{C}_{2}, \mathrm{C}_{3}, \mathrm{C}_{4}, \mathrm{O}_{6}\right)$ (rotation about the $\mathrm{C}_{3}-\mathrm{C}_{4}$ bond). Calculations were performed at the B2PLYP$\mathrm{D} 3 \mathrm{BJ} /$ aug-cc-pVTZ level of theory. The absolute energy of the lowest conformation is $\mathrm{E}=-346.95152$ Hartree. Dash lines indicate the minimum energy pathway shown in Figure 5.

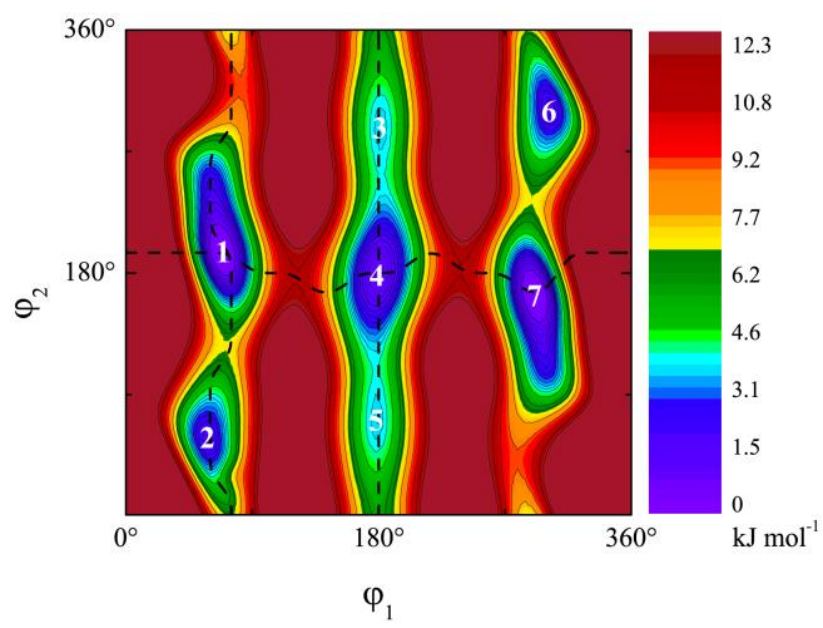


Figure 3: The trans conformers of methyl butyrate in order of their energies relative to that of the most stable conformer $(\mathbf{g} \pm, \mathbf{a})$ calculated at the MP2/6-311++G(d,p) level with and without zero point energy correction as well as at the B2PLYP-D3BJ/aug-cc-pVTZ level (for values see Table 1).

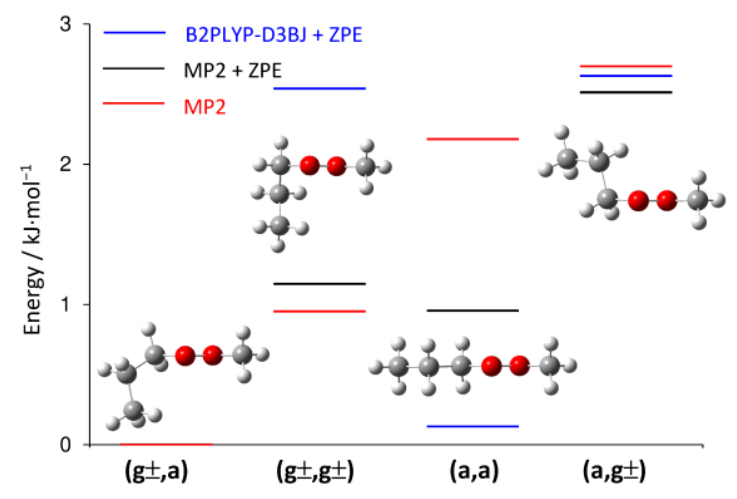

Figure 4: The CP-FTMW broad band scan over the 8-18 GHz region and a high-resolution measurement of the A species of the $3_{13} \leftarrow 202$ transition of conformer $(\mathbf{g} \pm, \mathbf{a})$ of methyl butyrate.

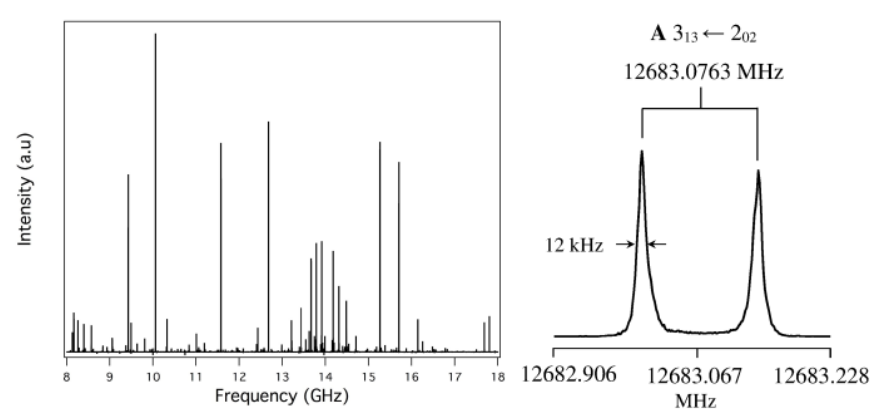


Figure 5: Presents three minimum-energy pathways along the 2D surface illustrated in Figure 2. a) A cut along $\varphi_{1}$ through the (a,a) (4) and (g士,a) (1 and 7) minima. b) A cut along $\varphi_{2}$ through the $(\mathbf{g} \pm, \mathbf{g} \pm)(2)$ and $(\mathbf{g} \pm, \mathbf{a})(1)$ minima. c) A cut along $\varphi_{2}$ through the (a,g \pm ) (3 and 5) and (a,a) (4) minima. The barrier heights are indicated in blue and in red the relative energies. The numbers in black indicate which minima we are referring according to Figure 2.
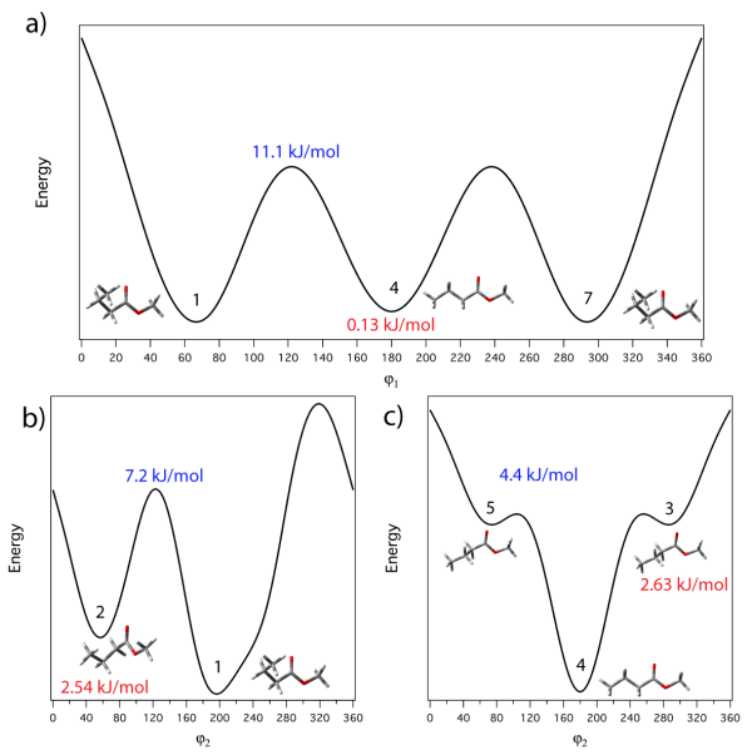